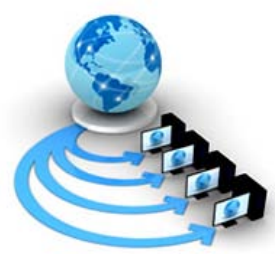

Volume 8, No. 9,November-December 2017

International Journal of Advanced Research in Computer Science

\title{
A QUIZZING SYSTEM FOR BLIND AND VISUALLY IMPAIRED CANDIDATES
}

\author{
Réginald Cherenfant Jasmin \\ University of the West Indies, \\ Trinidad and Tobago
}

\author{
Michael Hosein \\ University of West Indies, \\ Trinidad and Tobago
}

\begin{abstract}
A major obstacle confronting blind and visually impaired students in their education is the lack of ability to function independently when it comes to certain things like taking exams. This paper is about a voice-enabled quizzing system which will be used to assess the students of a university especially the blind or visually impaired. The system was built on top of the voice over IP (VoIP) technologies in conjunction with an Automatic Speech Recognition (ASR) engine used to transform a speech to its actual text format.Multiple Choice and True/False Question are the only types of question allowed for a quiz; and the interactions with the system are fully operated by voice commands by using a mobile phone, a SIP-enabled device or a SIP-based application to call a number associated to an Interactive Voice Response (IVR) provided with a Voice User Interface (VUI) to manage quizzes as a lecturer and to take quizzes as a student. After the completion of a quiz, the result is provided automatically to the student by audio and email. Immediately afterthe end date and time set for a quiz is elapsed, an email containing statistical data and the attendants' results is sent to the lecturer to analyze the performance of the students. However, the lecturer can review the quizzes results anytime by using the Voice User Interface.
\end{abstract}

\section{INTRODUCTION}

The wireless and communications technologies and mobile devices such as mobile phones, tablets, PDAs, laptop are considerably becoming available, cheaper and more convenient. From day to day new versions of mobile devices with additional applications and features such as SMS, GPS, NFC, Wi-Fi, Bluetooth, and Softphone are released and marketed frequently. Over the past ten years, the use of the mobile technologies grew considerably around the world since they involve distant and wireless communication between devices.

Out of a worldwide population of 6 billion, a total of 1.7 billion mobile phones are currently in use (Hashemi et al. 2011), however, it reveals that the access to mobile networks is available to $90 \%$ of the world population (Gedik et al. 2012).

These technologies are practically used in different sectors such as; education, health, and agriculture to solve problems, to enhance systems' performance and functionalities and to facilitate remote access to resources.End users are more comfortable to adopt the practices of wireless communication technologies over wired communication technologies since physical connections are not used during the communication session between endpoints. This highly enables mobility and helps to a rapid increase of the network coverage. Regardless any technical issues faced by the mobile technologies, they are massively adopted by researchers, educators, and educational institutions to expand the process of teaching and learning (Wu et al. 2012) since they allow distant teaching, learning and more interactions between lecturers and learners by using their personal mobile devices such as mobile phone, tablets, and notebooks; hence the concept of mobile learning or simply M-learning.

Mobile learning can be defined as the combination of the mobile technologies and human capabilities to make the teaching and learning process more flexible (Gedik et al. 2012). It allows the extension of the traditional classroom by creating and using mobile wireless-basedapplications to share course materials, to participate in class forum discussions and to take exams from any location, at any time (Hashemi et al. 2011).

Blindness and visual impairment are a severe problem around the world. These types of disability are most likely coming from sicknesses such as onchocerciasis, xerophthalmia, glaucoma, cataract, and trachoma (WHO 2017). In a study conducted in India by (Minassian and Mehra 1990), 3.8 million persons are becoming blind by cataract every year and (Thylefors et al. 1995) estimated that globally 38 million persons are blind and 110 million have low vision and are presenting a very high risk to become blind. People who are affected by these types of disability are very challenged during their educational training since they are lacking the ability to read and to write texts on regular paper without assistances of special machines or software.

This research paper proposes a lightweight, user-friendly and reliable quizzing system referred to as QuiVox, that can assist the blind and visually impaired students of a university in taking exams independently. The system was built with the use of the mobile technologies especially the Voice over IP technologies. The core of the systemconsists of a IP PBX(Asterisk) sever that is used to allow the User Agents such as SIP phones, SIP-based applications to register their SIP address on the PBX registry and to establish a media session with the customized IVR by dialing a specific extension number. When a call session is established with the customized IVR the IP PBX executes the instructions of the corresponding dial plan. The students can access the system only through a Voice User Interface associated to the customized IVR to take quizzes and review their results, while the lecturers can setup quizzes via a web Graphical User Interface (GUI). An Application Programming Interface(API) is available to allow interactions with third party systems. The Voice User Interface can be also used by the lecturers to record audio file for the questions of a quiz and to review statistical data.The system is operated by voice commands and it supports only Multiple Choice and True/False questions. 
Questions are delivered to thestudents in audio format and the answers are providedin the form of human voice which is transformed to its actual text format by an Automatic Speech Recognition(ASR) for further processing. Results is provided automatically to the student via audio and email right after the submission of the answers. The lecturer receives an email with statistical data and the students' scores to analyze their progression and performance.

\section{LITERATURE REVIEW}

With the rapid advancement of the information and communication technologies (TIC), researchers around the world have conducted many research studies to craft novel solutions on how to integrate these technologies in the educational systems to facilitate remote access to course materials, sharing ideas between students and lecturers, learning assessment and so on.

(Yengin et al. 2011) proposed an SMS-based system that could be used as a quiz tool to facilitate learning in the educational institutions. The system supports Multiple Choice Questions and enables an automated grading process. The questions of quiz were displayed inside the classroom on a presentation screen, in a handout or on a web site, and then the lecturer asked the students to answer the questions by sending SMS messages. The answers provided by the students were stored in a database, and real-time feedback was provided to them.

In the same study, the researcher proposed a classroom pooling system to allow students to participate in a survey during the class. The survey system was operated similarly to a clicker system, the questions were sent directly to the students' phone as an SMS message and they replied by resending an SMS. The results of the polling were displayed in real-time as bar chart graphs on a screen in the class.

The teachers in the department of Information Systems of ISCAP, which is an institution of accounting and management studies in Porto, Portugal, have been using the e-learning platform Moodle combined with the traditional assessment fashion for several years. Multiple-Choice Questions (MCQ) tests were implemented using Moodle quizzes tool to assess theoretical topics. However, this method was becoming more challenging to use when there was an evaluation of a practical topic. (Babo, Azevedo, and Suhonen 2015) developed a system in combination with the traditional Moodle assessment system to evaluate practical topics like management problem solving. The system uses a spreadsheet to collect the answers from the students and an automatic grading strategy.

(Fluck, Pullen, and Harper 2009) presented a new computerbased exam system that involved the customization of an open source live Ubuntu-based CD copied from the original operating system (OS) distribution. The questions' file is in Microsoft Word format and it is placed in a folder on the desktop. For security purpose and annoyance avoidance, certain components such as; Bluetooth, networking card and sound card were disabled to prevent data sharing, access to online resources and noise from the computers during the time of examination. Each candidate was assigned a copy of the customized Live Ubuntu CD to ran on their computer and to answer the questions. At the end of the examination, the candidates must re-save the file containing the exam's questions and answers provided with a new filename comprising their surname and identification number. When the students had completed their exams, they must close all opened software without switching or logging out the computer.

In a feasibility study conducted by (Dearnley, Haigh, and Fairhall 2008) in using mobile technologies to assess the students of the health and social care department of a nursing school in clinic practical settings; a PocketPCbased system was implemented and tested using a target group constituting of five lecturers and twenty-nine students. Each groupmember was issued a PocketPC containing the assessments documentations. Results of the tests and questionnaires survey conducted showed that students preferred the PocketPC-based system over the paper-based format. The main drawback of that system was the lack of synchronization mechanism between the mobile device and the University Diary system because the loss of the PocketPC would have resulted in the loss of the entire data stored.

(Hinkelman and Grose 2004) proposed a low-cost alternative system that schools teaching foreign languages could use to assess students when it comes to divide them into levels based on their ability in the taught languages. The system implemented improves the content management system referred to as Moodle to support audio-based listening/reading test.The type of question supported by the system was only Multiple-Choice Questions (MCQ) and each question was associated to an MP3 audio-file uploaded into the system database, which the test-takers could pause/stop/playas many times as they wished by keeping in mind that the test is timed.

An interesting system was proposed by (Motiwalla 2009), which consists of a voice enabling interactive system referred to as VòIS, accessible via mobile phones and allowed normal users and those with visual disabilities to interact in real-time with an online forums discussion system by using the voice-enabled technologies such as Automatic Speech Recognition (ASR) and Text-To-Speech (TTS). Following is a figure showing the architecture of VòIS:

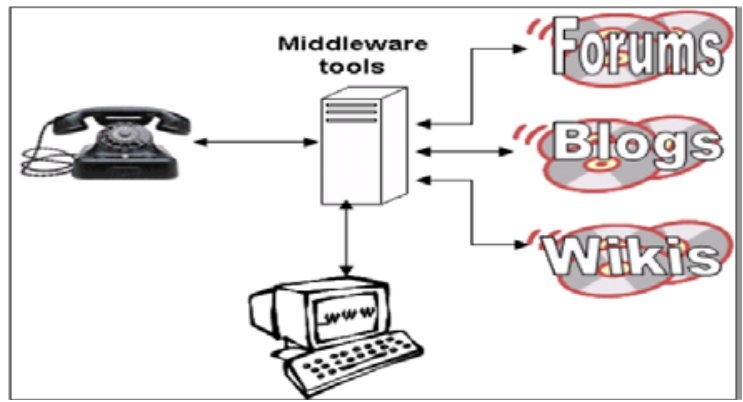

Figure 1: VòIS Architecture(Motiwalla 2009)

A solution was proposed by (Shahreza 2006) consisted of a secured SMS-based quizzing system. The system supported Multiple-Choice Questions and SMS on a mobile phone is used to take a quiz. The students received the quiz questions and answers to the questions from the instructor by SMS. After answering all the questions, the students received their grade and sent them to the instructor. The grades were secured using the steganography method within an SMS picture to prevent cheating by the students. 
A web-based quizzing application referred to as Pitalica has been developed and proposed by (Gamulin and Gamulin 2012) to assess students in the physics laboratory class at a university in Croatia. The quiz questions used multiple choice technique, which were chosen randomly from an available pool by the web application. Each question has a limited answering pre-set time based on its severity. To avoid cheating the sequence of the questions were varied. Students and instructors got the results of the tests within 45 days.

Another very interesting solution was developed by (Ţucă and Iftene 2017) to help geometry teaching. The proposed application called "Voice Geometry Painter" allows mathematics teachers and students to draw geometric shapes on a virtual board through a Voice User Interface by sending voice command like "draw <shape-name $><$ parameters $>$ ". The application used Speech Recognition technology to transform the users' voice commands to text commands before they were sent to the command parser component for further processing. The major purpose of the study was to provide persons with motion disabilities the opportunities to learn, manipulate and draw geometry shapes.

(Fujiyoshi and Fujiyoshi) carried out a new audio system that enables learning todisabled people and newly blind who have difficulties with reading print-format and Braille to take test exams during their education. The system uses a tablet PC and the document structure of the test problems was presented with icons or characters on a computer screen and on a Braille paper for the learning disabled or newly blind respectively. The blind system consisted of A3 tablet, a PC, an amplified speaker and a ten Key-Pad. A sheet of braille paper was placed on the A3 tablet, on which the structure of the document or figure was printed using a braille printer. The test-takers uses an electronic pen when taking exams to navigate into the document and listened to speech sound from any part of it and answered the questions accordingly.

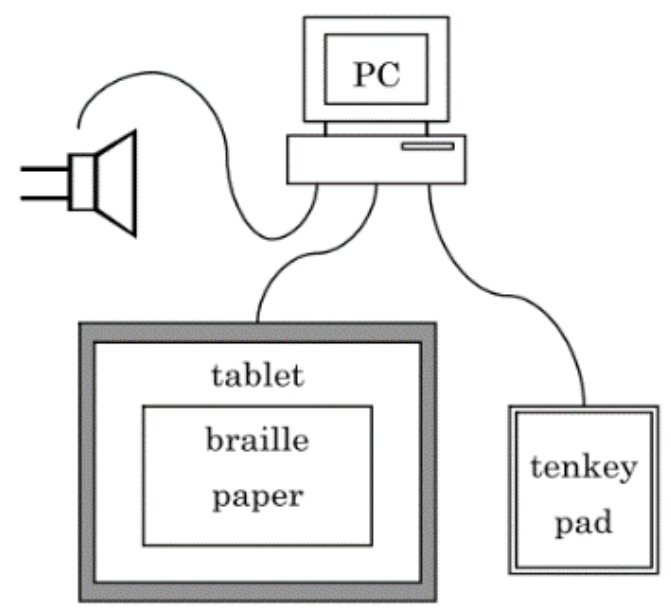

Figure 2 : Audio testing system for blind (Fujiyoshi and Fujiyoshi)
A quizzing system based on Bluetooth technology known as "BlueQ" was proposed by (Hosein and Bigram 2013). This system consisted of two main components, the server component, and the client component, where both are Android-based mobile application. However, the server application must be started by the lecturer to accept connections from multiple clients. Due to the single pairing constraint of Bluetooth technology, a queuing system was established on the server side to allow simultaneous connections from multiple clients to the server.The server must accept the request to start the communication with a client. The server component was controlled by the lecturer and allow him/her to manage connections, student's information, student's registration and quizzes. Multiple Choice Question (MCQ) was the type of quizzes question handled by BlueQ. The server component also provided automatic statistical reports in form of pie charts that allowed the lecturer to analyze results and kept track of student's performance. The client component was controlled by the students. It allowed him/her to connect to the server by turning on his/her Bluetooth phone card and send a connection request to the server. It also allowed students to review results of quizzes and attend available quizzes.

\section{SYSTEM DESIGN}

The system referred to as QuiVox, iscomposed of various components such as Database cluster, Application Server, SIP Proxy and load balancer, IP PBX (Registrar Server), Middleware, Mail Server, Media Gateway Server, Automatic Speech Recognition (ASR) engine and Text-toSpeech engine. These components are operated together to provide a reliable voice enabled quizzing service. QuiVoxis strictly dedicated to the sector of education, and it allows educators and learning institutions in providing a new technique to assess students especially those with visual disabilities. QuiVoxis accessible via the Voice User Interface(VUI) of a customized IVR by using mobile phones, SIP-enabled phones or SIP-based applications. The following diagram shows the architecture of the system in combination with the PSTN. 


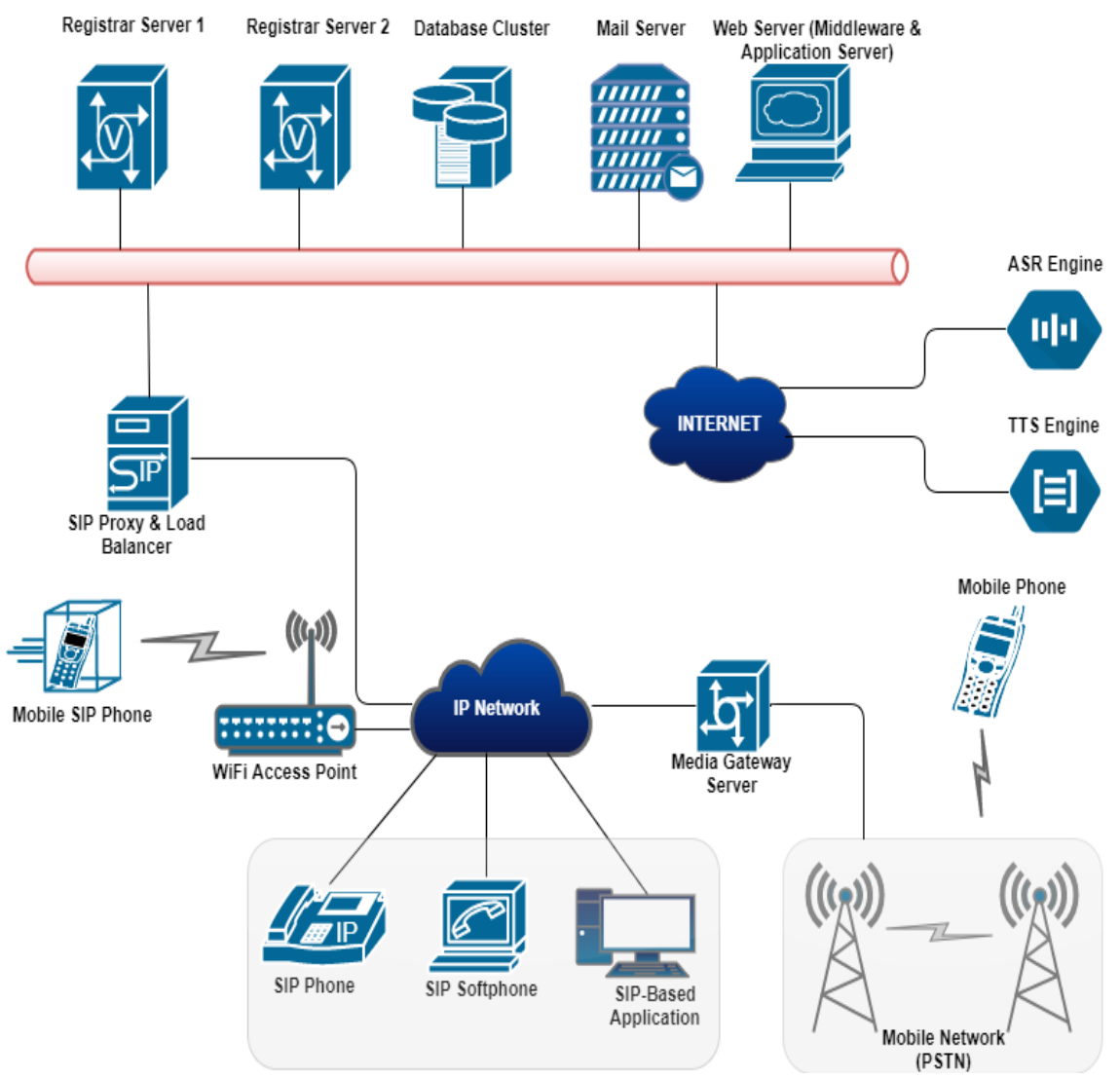

Figure 3: System Architecture

\section{COMPONENTS DETAILS}

This section presents briefly the different components of the system.

\section{A. Database Cluster}

The database cluster consists of two nodes and a database proxy communicating to each other. The cluster receives requests only through the database proxy, and forward them to the corresponding node. The nodes manage and store the application's data such as quiz data, student information data, lecturer information, and the call details records (CDR) data generated on the voice server. Each node contains a schema of the application database, which are replicated to each other

\section{B. Application Server}

The application server hosts the different applications and services that could be involved in the system cycle. These applications are The GUI web application, the middleware application, and the SOAP-based API.

\section{Middleware}

The Middleware is a TCP-based server application that enables communication between the VUI and the other applications and services such as the quiz application, the ASR engine, and the TTS engine. It has three main modules: the audio data parser, the commands analyzer, and the request/reply dispatcher. It receives the requests in the form of voice data from the users through the VUI. The requests are parsed and analyzed before they are forwarded to the correct service for further processing. The results of the requests are sent back to the users through the VUI as well.

D. IP PBX
The system is composed of two IP PBX controlled by a SIP proxy server. The IP PBXs have a registry of all the users (students and lecturers) SIP account. They allow the UAs such as SIP phones, SIP-based applications to register their SIP address on the PBX registry and to establish a media session with the IVR by calling a specific extension number. When a call session is established with the IVR the IP PBX executes the instructions of the corresponding dial plan.

\section{E. Mail Server}

The Mail Server allows the system to send out an email to the students after they submit the answers for each attended quiz, and to the lecturers when the date and time set for a quiz are over.

\section{F. Media Gateway Server}

This component on the network is used to provide interoperability between the VoIP network and the mobile network (PSTN), as they use a different type of signaling protocol to control a communication channel established between entities from both networks.

\section{G. Automatic Speech Recognition Engine}

The Automatic Speech Recognition engine transforms and converts the user's speeches collected from the VUI into their corresponding text format for further processing.

H. Text-To-Speech Engine

The Text-To-Speech engine converts a text into its corresponding audio version, to be played to the user through the VUI. 


\section{VOICE USER INTERFACE (VUI) PIPELINE}

The voice interface supplied by system uses a combination of three processes, thatare executed sequentially to provide its service. The following diagram shows the various stages executed during the treatment of a speech received through the VUI.

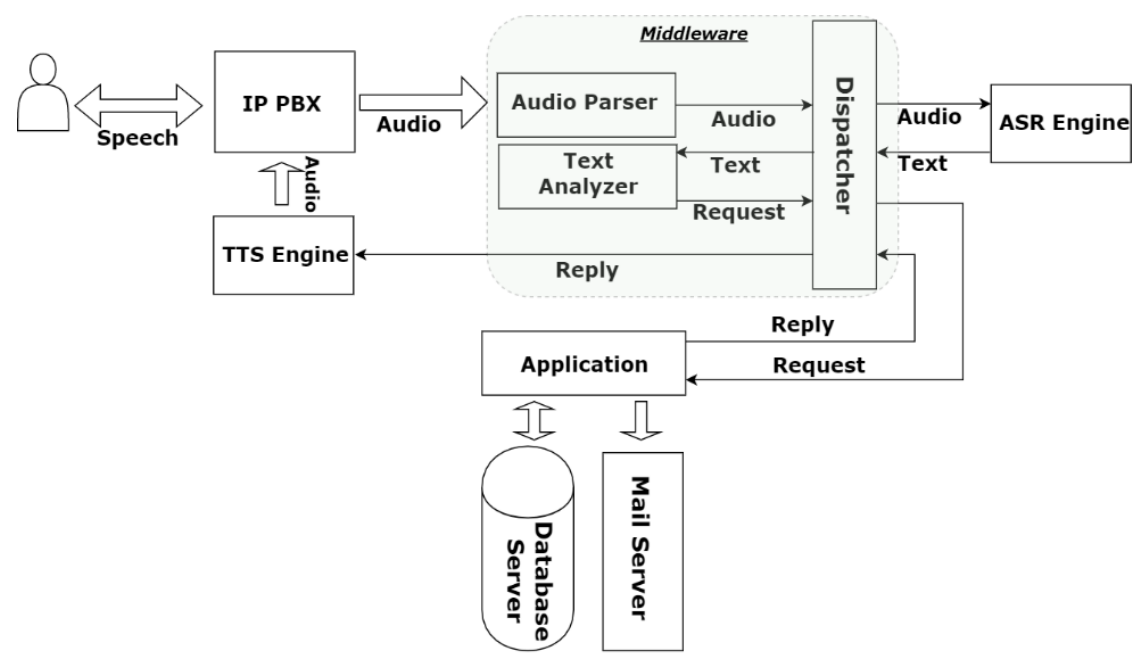

Figure 4: VUI Pipeline

\section{EXPERIMENTAL SETUP AND RESULTS}

The system architecture setup for the experiment consists a VoIP network with three virtual servers noted as VM1, VM2 and VM3, and two SIP-enabled softphones, which are 3CX v6 softphone (windows version) and a Zoiper mobile softphone (Android version). This architecture is used to deploy and setup the different tools and software components required to make the system operational for our test users' group.

The virtual environment setup in the experiment has been configured on a ThinkPad Edge Lenovo E530 Laptop supplied of the following specifications:

Operating System: Windows 7 Professional Edition 64-bit

Processor: Intel ${ }^{\circledR}$ Core(TM) i5-2500M CPU @ 2.50GHz, Dual Core

RAM: $10.0 \mathrm{~GB}$

Hard Drive Memory: 500 GB

Oracle VM VirtualBox Version 5.0.8 r103449 has been used as virtualization software manager on the laptop to create and to configure the different guest virtual machines and networks.

Network and computing resources are provided by the laptop to the different virtual components such as the virtual machines and the 3CX softphone.

A VirtualBox internal network has been setup to allow the communication between the guest machines, which are equipped with software componentssuch as Asterisk 11.25.1, MySQL Server 5.7.16, MySQL NDB Cluster 7.5.4,
OpenSIPS 2.3.1, 3CX and Zoiper softphone, Virtual-wifirouter-3.0.1.2 and JAVA 1.7.0_79 that are parts of the system setup.

Google Speech Recognition and Text-ToSpeechAPIshave been used to handle the speech recognition and the transformation of a text to audio. Both are cloud-based REST API provided by Google to transform a speech to its actual text meaning vice-versa. Google uses a very strong neural network and machine learning algorithms behind these APIs. A range of factors such as latency supported language, and accuracy was taking into consideration to make choice of these API over others. They are widely used in voice command based system since they support:

- $\quad$ More than 80 languages.

- $\quad$ Real Time speech recognition processing.

- Recognition of prerecorded audio from mobile device.

- $\quad$ Noise filtering.

- High accuracy.

- Very low cost of usage.

- Pay-as-Go payment method.

Google Mail Server: is a cloud-based Mail server, it was used to send out emails to the users after the completion of a quiz exam. a Gmail account was created and used to authenticate to the Mail server online using Simple Transfer Protocol(SMTP).

The following diagram shows the architecture of the experimental setup that has been done. 


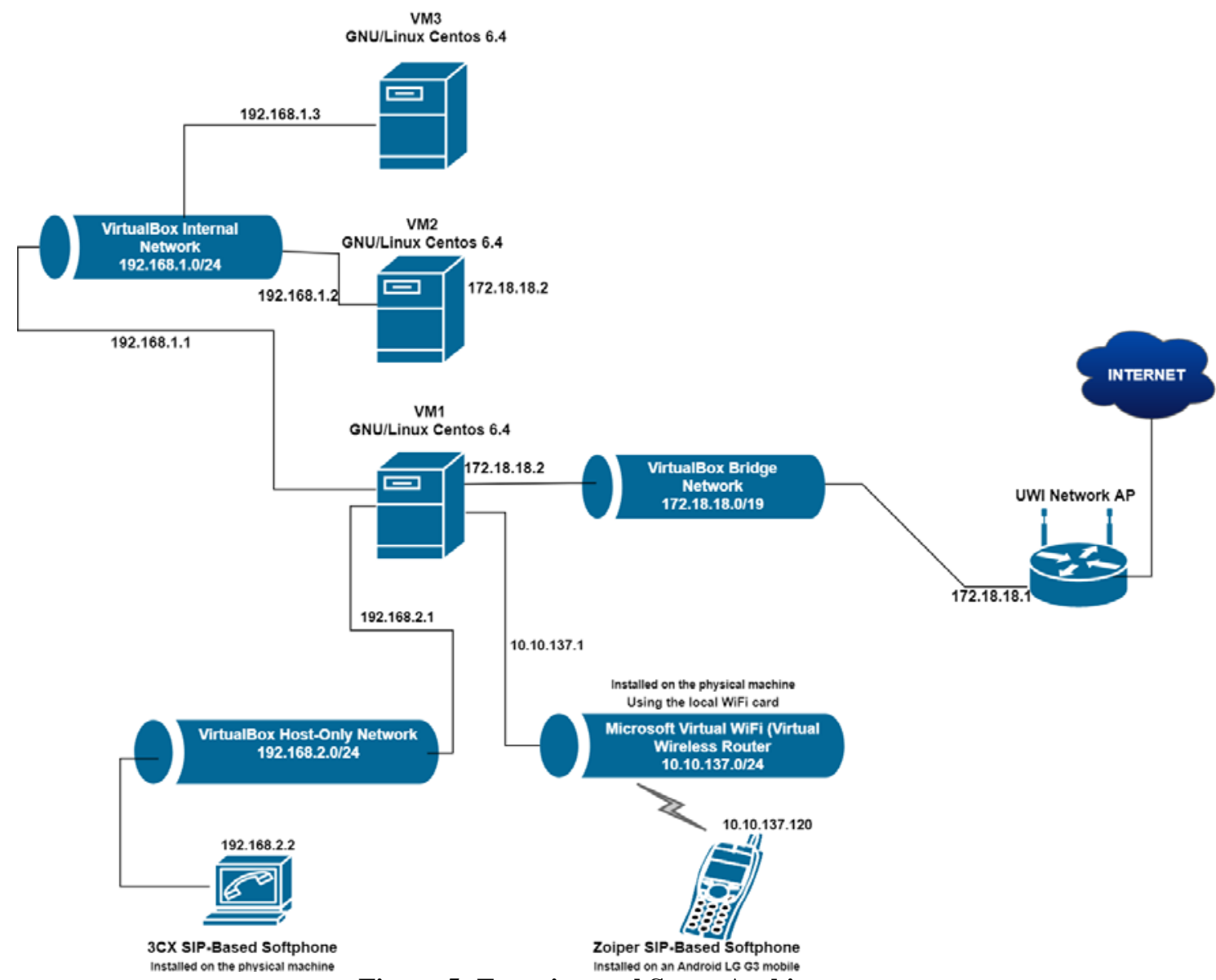

Figure 5: Experimental Setup Architecture

The experiment was implemented and tested at the University of the West Indies, St Augustine campus. A quiz was setup and stored in the system database. The subject used was "Distributed Computers Systems" which is taught in the MsC program in Computer Sciences at the university.The VUI was tested by fifteen (15) users. A SIP account was created for each of these users on the media servers to allows them to register at the registrar servers (IP PBX) before they can access the VUI. To access the VUI, the successfully registered users dialed the extension number "123"

\begin{tabular}{|llll}
\hline FirstName & LastVame & Email & Type \\
\hline Micheal & Hosein & gilias@avahoo.ff & LECTURER \\
\hline
\end{tabular}

\begin{tabular}{|ll|}
\hline Subjectlvame & SubjectCode \\
\hline Distributed Comouter Svstems & 6601 \\
Advanced Comouter Network & 6401 \\
Parallel Databases & 6800 \\
\hline
\end{tabular}

\begin{tabular}{|llllll}
\hline QuizCode & SubjectName & Question & CorrectAnswer & TotalMark & Rank \\
\hline 6000 & Distributed Comouter Svstems & The kev difference between the two svstems is... & true & 2 & 1 \\
6000 & Distributed Comouter Svstems & A svstem in which the comoonents of an inform... & A & 2 & 2 \\
6000 & Distributed Comouter Svstems & Which of the followino define the acolication arc... B & 2 & 3 \\
6000 & Distributed Comouter Svstems & A server that hosts services for e-mail. calenda... & C & 2 & 4 \\
6000 & Distributed Comouter Svstems & Which technioue alocates different rows in a ta... & A & 2 & 5
\end{tabular}

Figure 6: Quiz Setup

The following figure shows the configuration of the SIP account configured for the "user2" on the 3CX softphone installed on the physical machine (the laptop). The registration of the User Agent Client (3CX Softphone) is also shown on the registrar server after the configuration setup is done. 

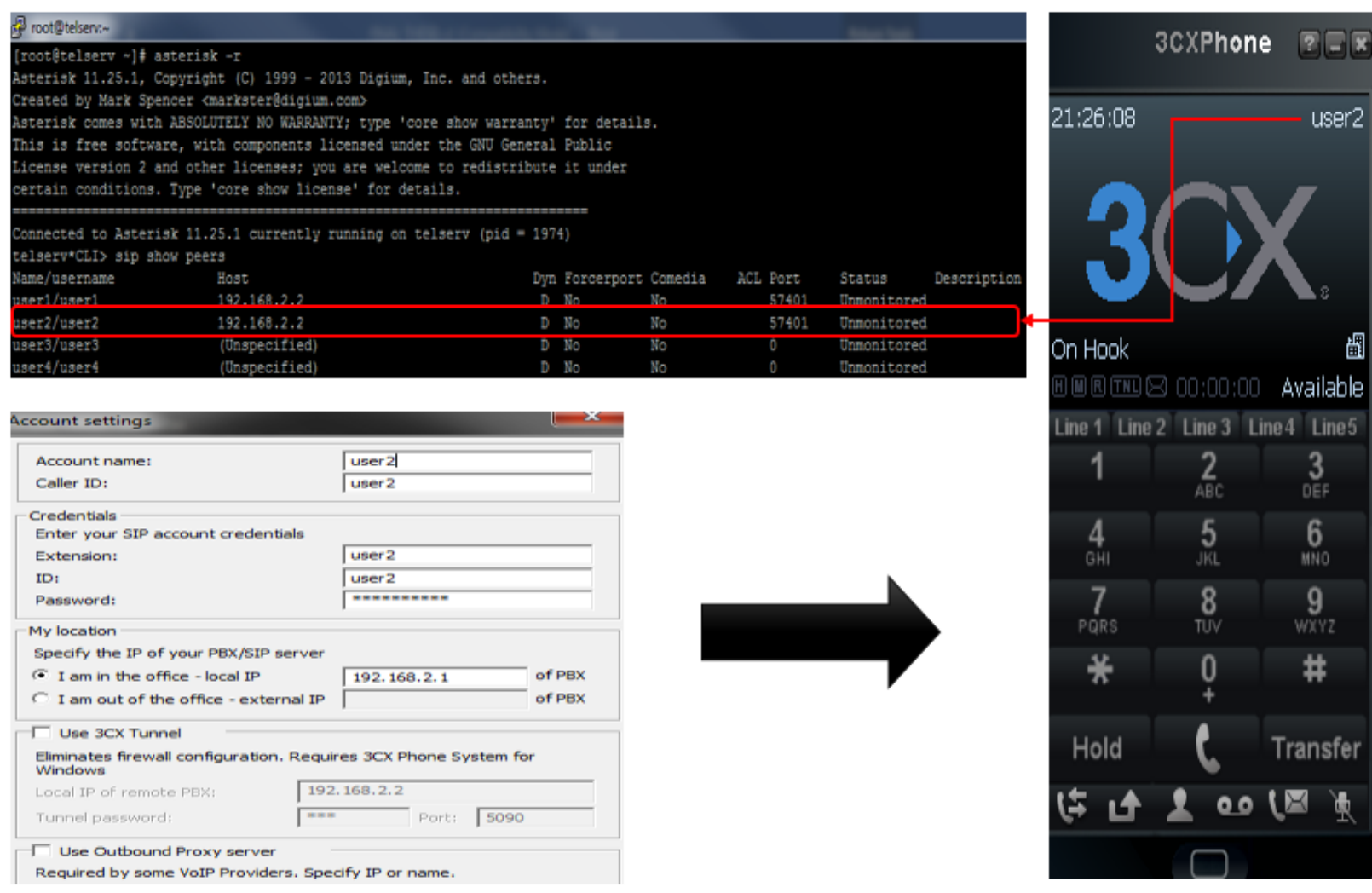

Figure 7: SIP Client Configuration and Registration

The users dialed the extensions number "123" to access the quiz and answer questions by voice. The answers provided by the students were stored into the database to ensure that

\begin{tabular}{|c|c|c|}
\hline Student_FullName & Question & Answer \\
\hline \multirow[t]{5}{*}{ Reginald Cherenfant Jasmin } & Question 1 & TRUE \\
\hline & Question 2 & A \\
\hline & Question 3 & B \\
\hline & Question 4 & C \\
\hline & Question 5 & A \\
\hline Student_FullName & Question & Answer \\
\hline \multirow[t]{5}{*}{ Kemar Celestin } & Question 1 & TRUE \\
\hline & Question 2 & c \\
\hline & Question 3 & B \\
\hline & Question 4 & C \\
\hline & Question 5 & B \\
\hline
\end{tabular}

After the completion of the quiz, the student received automatically his/her score by audio and the following email with the answers submitted and its total score as well.
Figure 8: Quiz Answers Provided

they are available for review at any time and to ensure there are proper records for all quizzes. 


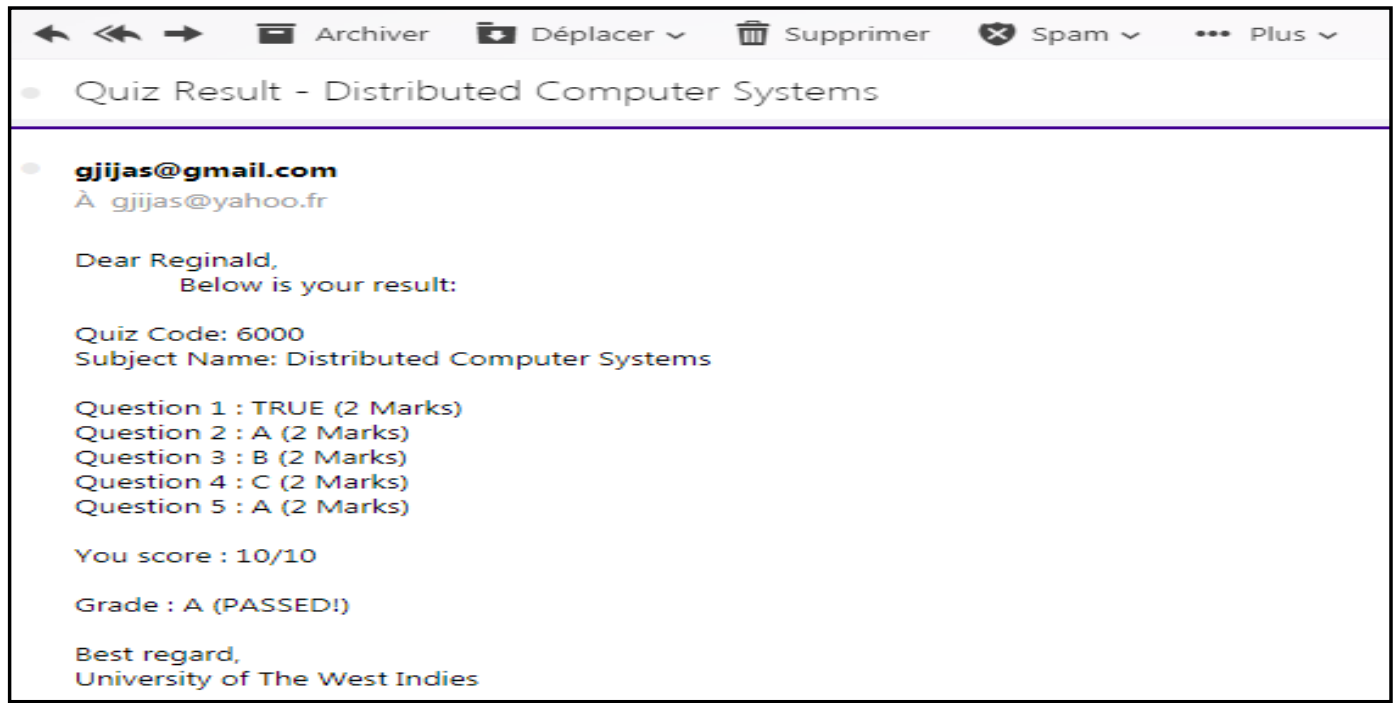

Figure 8: Student Quiz Result Email

After theenddate and time set for the quiz is elapsed, the lecturer received automatically the email containing the students' score and the percentage of failure and pass for each question.

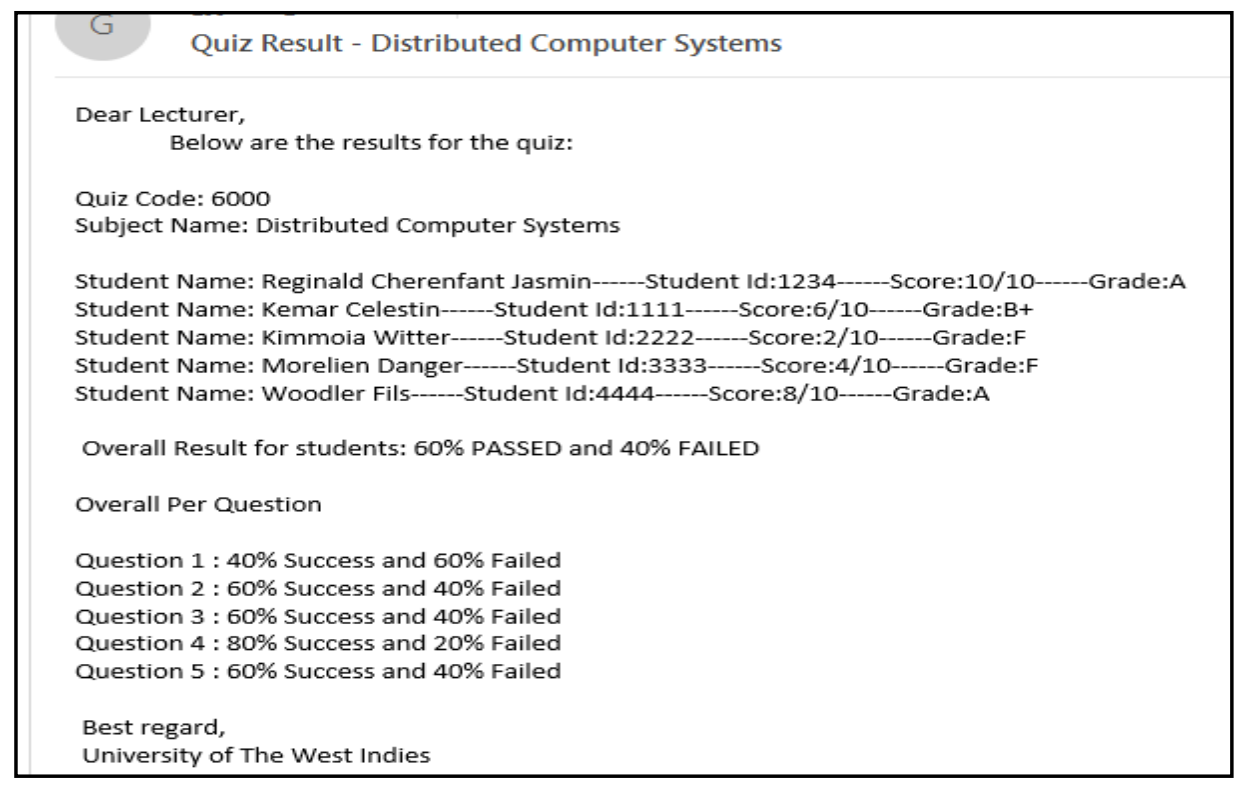

Figure 9 :Lecturer Quiz Results Email

\section{CONCLUSION}

This research study was focused on the use of mobile technologies in the area of education, with a specific aim to develop a multiple choice and true/false question supported quizzing system using an interactive voice response built on VoIP technologies. The system was conceived and developed especially for the blind and visually impaired learners to provide them with the capability of taking quiz independently through a user's Voice Command Interface during their education.

The system was built with the combination of the VoIP, Automatic Speech Recognition (ASR) and Text-to-Speech (TTS) technologies. It supports only Multiple Choice and True/False Questions

A prototype of the system was developed and deployed on an emulated testbed, and was tested with a group of 15 students at the University of the West Indies (UWI)-St. Augustine Campus. The test was completely successful and the results found were very satisfactory since they met the initial expectations.

The system currently can only facilitate multiple choice and true/false questions. The system can be further enhanced in the future to accommodate other types of question such as short answer, fill in the blank and matching. It also only supports the English language, but in the future, it can be developed to support multiple languages.

\section{REFERENCES}

1. Babo, Rosalina Bessa, Ana Isabel Azevedo, and Jarkko Suhonen. 2015. "Students' Perceptions about Assessment Using an e-Learning Platform." 244-246.

2. Dearnley, Chris, Jackie Haigh, and John Fairhall. 2008. "Using mobile technologies for assessment and learning in practice settings: A case study." Nurse Education in $\begin{array}{lcl}\text { Practice } & 8 & \text { (3):197-204. }\end{array}$ doi: http://dx.doi.org/10.1016/j.nepr.2007.07.003.

3. Fluck, Andrew, Darren Pullen, and Colleen Harper. 2009. "Case study of a computer based examination 
system." Australasian Journal of Educational Technology.

4. Fujiyoshi, Mamoru, and Akio Fujiyoshi. "A New Audio Testing System for the Newly Blind and the Learning Disabled to Take the National Center Test for University Admissions."

5. Gamulin, J., and O. Gamulin. 2012. "The application of web-based formative quizzes in laboratory teaching in higher education environment." 2012 Proceedings of the 35th International Convention MIPRO, 21-25 May 2012.

6. Gedik, Nuray, Arzu Hanci-Karademirci, Engin Kursun, and Kursat Cagiltay. 2012. "Key instructional design issues in a cellular phone-based mobile learning project." Computers \& Education 58 (4):1149-1159. doi: http://dx.doi.org/10.1016/j.compedu.2011.12.002.

7. Hashemi, Masoud, Masoud Azizinezhad, Vahid Najafi, and Ali Jamali Nesari. 2011. "What is Mobile Learning? Challenges and Capabilities." Procedia - Social and Behavioral Sciences 30:2477-2481. doi: http://dx.doi.org/10.1016/j.sbspro.2011.10.483.

8. Hinkelman, Don, and Timothy Grose. 2004. "Placement testing and audio quiz-making with

9. open source software." ResearchGate.

10. Hosein, Michael, and Laura Bigram. 2013. "AN EDUCATIONAL BLUETOOTH QUIZZING APPLICATION IN ANDROID." International Journal of Wireless \& Mobile Networks (IJWMN) 5.

11. Minassian, D C, and V Mehra. 1990. "3.8 million blinded by cataract each year: projections from the first epidemiological study of incidence of cataract blindness in India." British Journal of Ophthalmology 74 (6):341343. doi: 10.1136/bjo.74.6.341.

12. Motiwalla, L. F. 2009. "A Voice-Enabled E-Learning Service (V\&\#x0F2;IS) Platform." 2009 Fifth International Conference on Networking and Services, 20-25 April 2009.

13. Shahreza, M. S. 2006. "M-Quiz by SMS." Sixth IEEE International Conference on Advanced Learning Technologies (ICALT'06), 5-7 July 2006.

14. Thylefors, B., A.-D. Negrel, R. Pararajasegaram, and K.Y. Dadzie. 1995. Global data on blindness. World Health Organization.

15. Țucă, L. P., and A. Iftene. 2017. "Speech recognition in education: Voice geometry painter application." 2017 International Conference on Speech Technology and Human-Computer Dialogue (SpeD), 6-9 July 2017.

16. WHO. 2017. "Causes of blindness and visual impairment." World health Organization.

17. Wu, Wen-Hsiung, Yen-Chun Jim Wu, Chun-Yu Chen, Hao-Yun Kao, Che-Hung Lin, and Sih-Han Huang. 2012. "Review of trends from mobile learning studies: A meta-analysis." Computers \& Education 59 (2):817-827. doi: http://dx.doi.org/10.1016/j.compedu.2012.03.016.

18. Yengin, Ilker, Adem Karahoca, Dilek Karahoca, and Hüseyin Uzunboylu. 2011. "Is SMS still alive for education: Analysis of educational potentials of SMS technology?" Procedia Computer Science 3:1439-1445. doi: http://dx.doi.org/10.1016/j.procs.2011.01.027. 